\title{
Case Report \\ Long-Term Survival in Locally Advanced KRAS Wild-Type Pancreatic Adenocarcinoma
}

\author{
Marion Alhenc-Gelas $\left(\mathbb{D},{ }^{1}\right.$ Romain Cohen $(\mathbb{D}),{ }^{1}$ Pascale Cervera, \\ Jean-Christophe Vaillant, ${ }^{3}$ and Thierry André ${ }^{1}$ \\ ${ }^{1}$ Sorbonne Université, Department of Medical Oncology, AP-HP, Hôpital Saint-Antoine, F-75012 Paris, France \\ ${ }^{2}$ Sorbonne Université, Department of Pathology, AP-HP, Hôpital Saint-Antoine, F-75012 Paris, France \\ ${ }^{3}$ Sorbonne Université, Department of Digestive and Hepatobiliary Surgery, AP-HP, Pitié-Salpêtrière Hospital, F-75013 Paris, France
}

Correspondence should be addressed to Romain Cohen; romain.cohen@aphp.fr

Received 27 April 2019; Accepted 12 June 2019; Published 10 July 2019

Academic Editor: Chia-Tung Shun

Copyright (c) 2019 Marion Alhenc-Gelas et al. This is an open access article distributed under the Creative Commons Attribution License, which permits unrestricted use, distribution, and reproduction in any medium, provided the original work is properly cited.

\begin{abstract}
Pancreatic adenocarcinoma remains a cancer associated with a poor prognosis. For locally advanced pancreatic cancer (LAPC), median overall survival is approximately 16 months. Here we report the case of a 52-year-old LAPC patient treated with chemotherapy followed by chemoradiotherapy that was associated with a 14-year complete remission. A peritoneal relapse was then observed and chemotherapy was undergone until the patient died of infectious complications, 17 years after his diagnosis. The tumor was found KRAS, TP53, BRCA1, and BRCA2 wild-type. This KRAS wild-type LAPC-long survivor case report emphasizes the need to develop molecular approaches to predict LAPC patients' prognosis.
\end{abstract}

\section{Introduction}

Pancreatic adenocarcinoma (PC) is the fifth leading cause of cancer death and the second leading digestive cancer in incidence in Western countries, with a 5-year survival rate of less than 5\% [1,2]. For locally advanced PC (LAPC), median overall survival (OS) is approximately 16 months [3]. Here we report the case of long survivor of KRAS wild-type LAPC who benefited from a therapeutic strategy combining cytotoxic agents and radiotherapy.

\section{Case Report}

On July 2000, a 52-year-old man presented a loss of weight associated with dyspepsia. Ultrasonography showed a $4 \mathrm{~cm}$ mass in the head of the pancreas infiltrating biliary ducts, portal vein and an involvement of superior mesenteric artery greater than $180^{\circ}$. No metastatic dissemination was found. Pathological examination of the tumor revealed a moderately differentiated tubular pancreatic adenocarcinoma showing eosinophilic abundant cytoplasm and vesicular nucleus with a clear nucleolus. The patient previously received 10 cycles of a regimen combining gemcitabine $1,000 \mathrm{mg} / \mathrm{m}(2)$ as a 10 $\mathrm{mg} / \mathrm{m}^{2} / \mathrm{min}$ infusion on day 1 and oxaliplatin $100 \mathrm{mg} / \mathrm{m}(2)$ as a 2-hour infusion on day 2 every 2 weeks [4]. Tumor stability was observed while CA19.9 level decreased from $147 \mathrm{U} / \mathrm{mL}$ to $47 \mathrm{U} / \mathrm{mL}$. In March 2001, chemoradiotherapy was performed (45 Gy with a 10 Gy boost associated with 5-fluorouracil) and resulted in a radiological tumoral stability, with a normalized CA19.9 level.

Between April 2001 and May 2015, no tumor recurrence was detected. During this period, the patient presented several episodes of hematemesis, attributed to severe ulcerated bulbitis. The bulbitis was likely a side-effect of radiotherapy.

In May 2015, a disease recurrence was observed with jaundice due to an extrinsic obstruction of the common bill duct. Endoscopic biliary catheterism failed and eventually a surgical biliojejunal derivation was performed; peritoneal involvement was observed during the procedure. Chemotherapy was initiated in September 2015, with 5FU and oxaliplatin (FOLFOX regimen). Oxaliplatin was stopped after 7 cycles because of neuropathy and irinotecan was introduced after normalization of bilirubin for 5 more cycles until January 2016, followed with maintenance treatment by 
capecitabine until June 2016. At this time, disease remained stable with peritoneal diffuse infiltration and biliary duct dilatation.

Then, the patient benefited from a 12-month therapeuticfree interval. A diffuse lymph node tumor progression was detected in July 2017. A combination of capecitabine and oxaliplatin was initiated. Confirmed stable disease was observed and the treatment was withheld in December 2017 because of infectious complications. From January to March 2018, the patient presented several episodes of angiocholitis related to an anastomotic hepaticojejunal stenosis. A percutaneous biliary derivation was successfully performed with adapted antibiotherapy. Nevertheless the patient presented a new septic episode which revealed the appearance of multiples intrahepatic abscesses. CT-scans conducted at this period did not reveal any tumoral progression, but a neoplastic cholangitis could not be excluded from the causes of the biliary obstruction. Unfortunately in March 2018 the patient finally died of this medically challenging infectious complication.

An exploratory molecular analysis was performed on patient's tumor sample. No mutation was detected in PIK3CA, $B R A F, B R C A 1$, and BRCA 2 genes, neither in KRAS and TP53 genes. No loss of MMR protein expression was observed (i.e., mismatch repair proficiency).

\section{Discussion}

Here we report the case of a very long survivor of LAPC with KRAS wild-type mutational status. Long-term PC survivors are poorly characterized, with few described prognostic clinical, biological, or molecular parameters [5].

The patient was treated with chemoradiotherapy after induction chemotherapy. This strategy has not been demonstrated to improve neither OS (15.2 versus 16.5 months, hazard ratio (HR) 1.03, $\mathrm{p}=0.83$ ) nor progression-free survival (HR $0.78, \mathrm{p}=0.06)$ in the LAP07 phase III study but it enhances median delay to treatment reintroduction (6.1 versus 3.7 months, 95\% confidence interval (95\%CI) 4.8 - 7.0 months, $\mathrm{p}=0.02$ ) [3]. Based on this trial, a prognostic nomogram for LAPC patients with 5 independent prognostic factors of OS has been designed: age, pain, tumor size, albumin, and CA199. The PROLAP score dichotomizes LAPC patients into three prognosis groups with median OS of 15.4, 11.7, and 8.5 months $(\mathrm{p}<0.0001)$ [6]. According to the nomogram, the predicted median survival time of our 17-year LAPC survivor would have been about 14 months (95\%CI 11.75-15.5) and the 4year survival probability about 4\% (95\%CI 2-9). Nevertheless LAP07 study brought out the existence of long survivors after chemoradiotherapy who experience long remission periods without antitumor treatments.

Several genetic features have also been identified as prognosis biomarkers of PC patients. In 2012, an association was suggested between the number of mutated oncogenes and survival [7]. Focusing on the 4 main oncogenic drivers in PC (KRAS, CDKN2A, SMAD4, and TP53), a statistically significant association was found between the number of altered genes ( 1 or 2 versus 3 or 4$)$ and OS $(p=0.041)$ in a cohort of 79 patients. It is worth noting that these genetic alterations are harbored by the vast majority of PC (> 90\% for KRAS and CDKN2A, 75\% for TP53 and 55\% for SMAD4) $[8,9]$. Nevertheless the prognostic impact of KRAS mutation remains a matter of debate. Recently, Bournet and colleagues did not find KRAS mutational status as an OS prognostic factor, even if KRAS codon 12 mutation was significantly associated with a worse prognosis [10]. Similarly, KRAS mutation was not associated with OS in another cohort of 178 PC patients [11].

Interestingly KRAS wild-type status might be a predictive factor of response to EGFR inhibitors. In a phase IIb randomized trial evaluating first-line gemcitabine with or without nimotuzumab (a humanized monoclonal antibody acting like an EGFR inhibitor), 12 -month OS rate was $53.8 \%$ versus $15.8 \%$ in the KRAS wild-type population (HR $0.32,95 \% \mathrm{CI}$ $0.13-0.84, \mathrm{p}=0.026)$. It is worth noting that the proportion of KRAS wild-type patients in this study was surprisingly much higher than that usually described in literature $(26.5 \%$ in the combination arm and $41.7 \%$ in the gemcitabine arm) [12]. This result suggests that EGFR inhibitors might be efficient in first-line treatment for KRAS wild-type advanced PC patients.

\section{Conclusion}

Our case report emphasizes the need to develop molecular approaches in the field of PC to predict the patients' prognosis with more accuracy and to promote personalized therapeutic strategies.

\section{Consent}

Informed consent was obtained for this case report.

\section{Conflicts of Interest}

The authors declare that they have no conflicts of interest.

\section{Authors' Contributions}

All authors contributed equally to the manuscript. Romain Cohen is the article guarantor.

\section{References}

[1] A. Jemal, R. Siegel, J. Xu, and E. Ward, "Cancer statistics, 2010," CA: A Cancer Journal for Clinicians, vol. 60, no. 5, pp. 277-300, 2010.

[2] J. Ferlay, I. Soerjomataram, R. Dikshit et al., "Cancer incidence and mortality worldwide: sources, methods and major patterns in GLOBOCAN 2012," International Journal of Cancer, vol. 136, no. 5, pp. E359-E386, 2015.

[3] P. Hammel, F. Huguet, J.-L. Van Laethem et al., "Effect of chemoradiotherapy vs chemotherapy on survival in patients with locally advanced pancreatic cancer controlled after 4 months of gemcitabine with or without erlotinib the LAP07 randomized clinical trial," Journal of the American Medical Association, vol. 315, no. 17, pp. 1844-1853, 2016.

[4] C. Louvet, T. André, G. Lledo, P. Hammel, H. Bleiberg, C. Bouleuc et al., "Gemcitabine combined with oxaliplatin in 
advanced pancreatic adenocarcinoma: final results of a GERCOR multicenter phase II study," Journal of Clinical Oncology, vol. 20, no. 6, pp. 1512-1518, 2002.

[5] J. H. Jo, M. J. Chung, J. Y. Park et al., "Clinical characteristics of long-term survivors of inoperable pancreatic cancer," Pancreas, vol. 43, no. 7, pp. 1022-1031, 2014.

[6] D. Vernerey, F. Huguet, A. Vienot et al., "Prognostic nomogram and score to predict overall survival in locally advanced untreated pancreatic cancer (PROLAP)," British Journal of Cancer, vol. 115, no. 3, pp. 281-289, 2016.

[7] S. Yachida, C. M. White, Y. Naito et al., "Clinical significance of the genetic landscape of pancreatic cancer and implications for identification of potential long-term survivors," Clinical Cancer Research, vol. 18, no. 22, pp. 6339-6347, 2012.

[8] A. F. Hezel, A. C. Kimmelman, B. Z. Stanger, N. Bardeesy, and R. A. Depinho, "Genetics and biology of pancreatic ductal adenocarcinoma," Genes \& Development, vol. 20, no. 10, pp. 1218-1249, 2006.

[9] T. Tatarian and J. M. Winter, "Genetics of pancreatic cancer and its implications on therapy," Surgical Clinics of North America, vol. 96, no. 6, pp. 1207-1221, 2016.

[10] B. Bournet, F. Muscari, C. Buscail et al., "KRAS G12D mutation subtype is a prognostic factor for advanced pancreatic adenocarcinoma," Clinical and Translational Gastroenterology, vol. 7, article e157, 2016.

[11] M. Haas, S. Ormanns, S. Baechmann et al., "Extended RAS analysis and correlation with overall survival in advanced pancreatic cancer," British Journal of Cancer, vol. 116, no. 11, pp. 1462-1469, 2017.

[12] B. Schultheis, D. Reuter, M. P. Ebert et al., "Gemcitabine combined with the monoclonal antibody nimotuzumab is an active first-line regimen in KRAS wildtype patients with locally advanced or metastatic pancreatic cancer: a multicenter, randomized phase IIb study," Annals of Oncology, vol. 28, no. 10, pp. 2429-2435, 2017. 


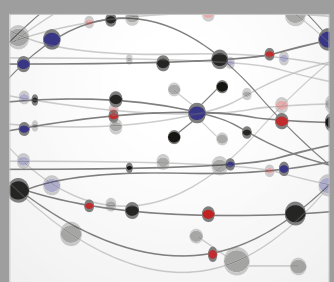

The Scientific World Journal
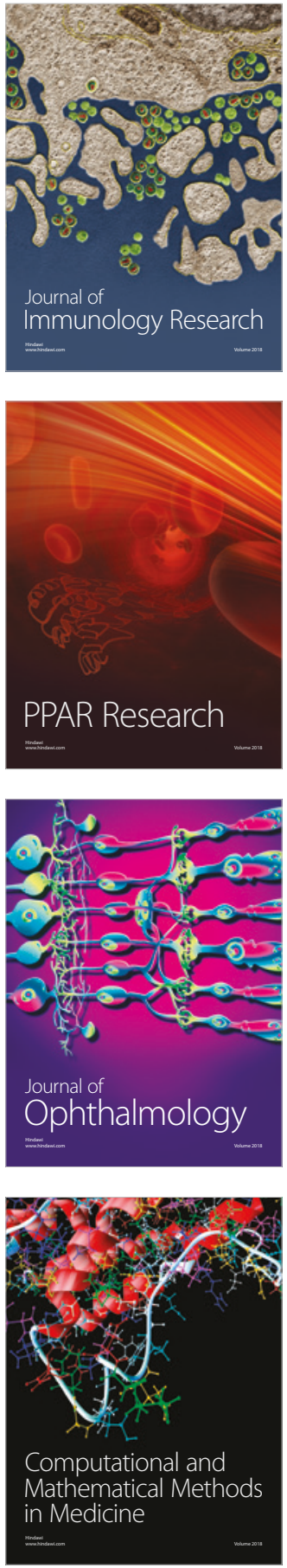

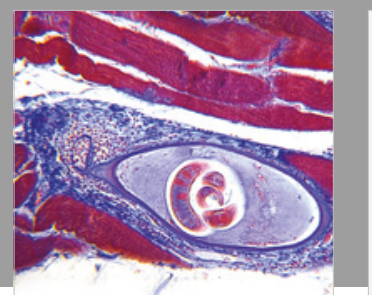

Gastroenterology Research and Practice

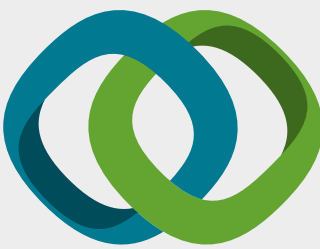

\section{Hindawi}

Submit your manuscripts at

www.hindawi.com
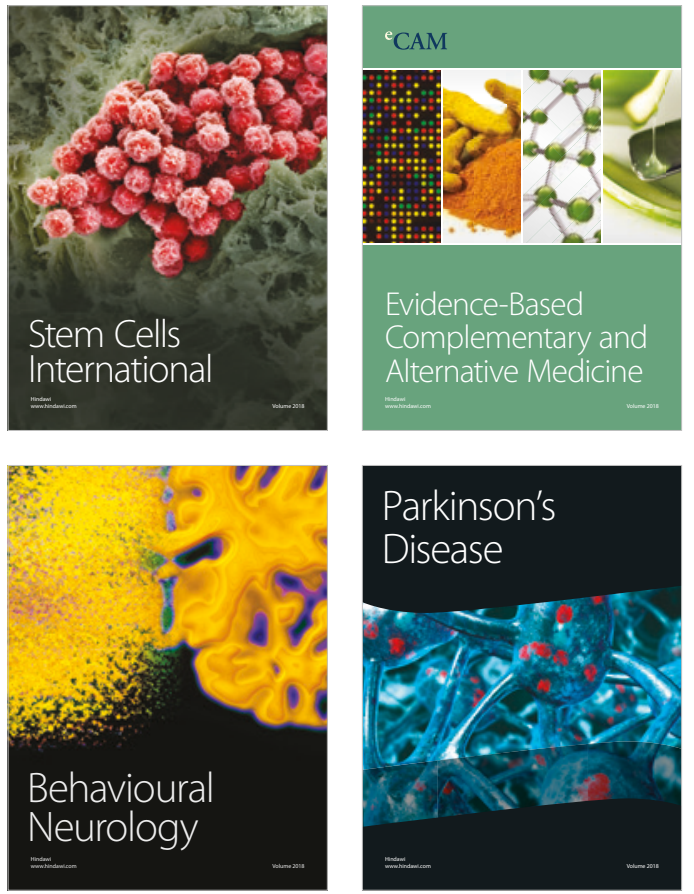

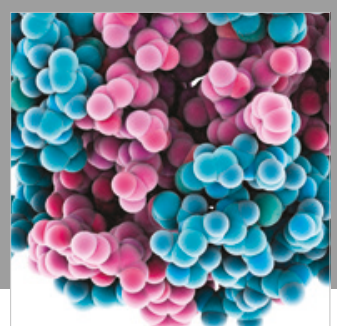

ournal of

Diabetes Research

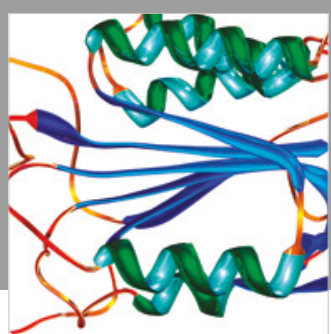

Disease Markers
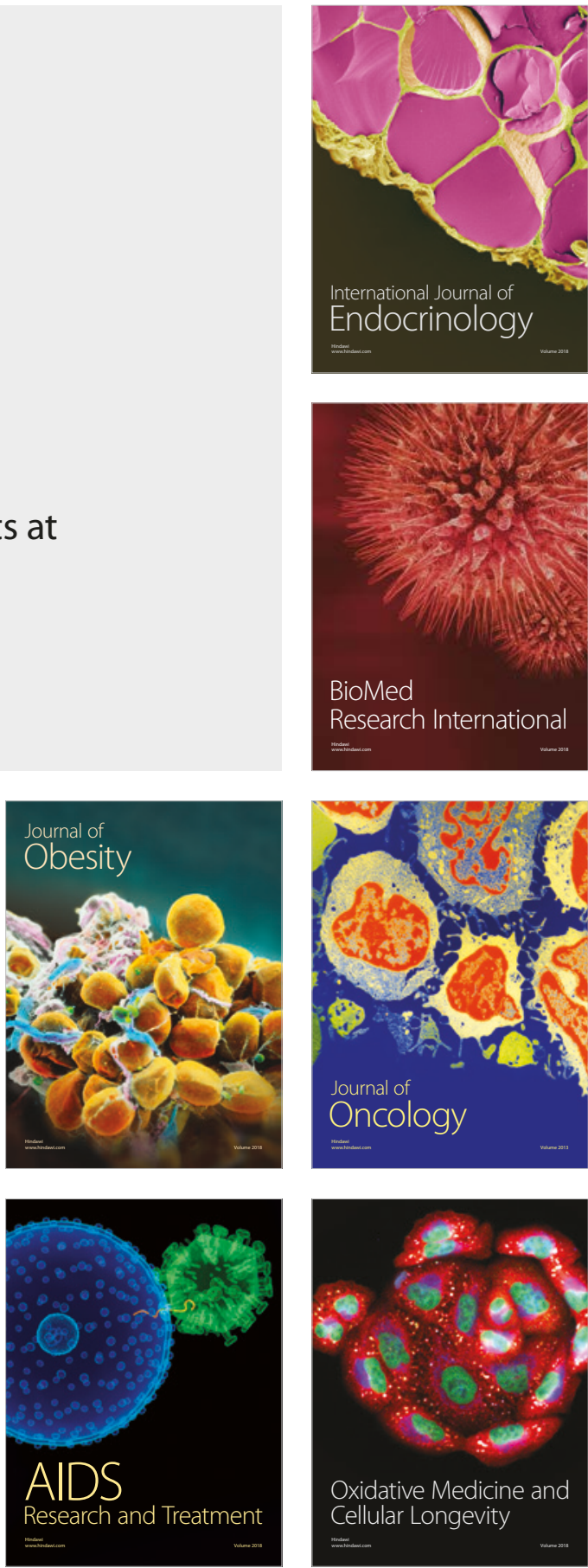